\title{
LIGEGFR: SPATIAL GRAPH EMBEDDING AND MOLECULAR DESCRIPTORS ASSISTED BIOACTIVITY PREDICTION OF LIGAND MOLECULES FOR EPIDERMAL GROWTH FACTOR RECEPTOR ON A CELL LINE-BASED DATASET
}

\author{
Puri Virakarin ${ }^{1, \dagger}$ \\ puri_v@kvis.ac.th \\ ๑ Jiramet Kinchagawat ${ }^{2}$ \\ jiramet.kin@kaist.ac.kr \\ Treephop Saeteng ${ }^{2}$ \\ treephops_pro@vistec.ac.th \\ (1) Naravut Suvannang ${ }^{2, *}$ \\ naravuts_pro@vistec.ac.th
}

(1) Natthakan Saengnil ${ }^{1, \dagger}$

nuttagun_s@kvis.ac.th

\author{
(1) Bundit Boonyarit ${ }^{2, \dagger}$ \\ bundit.b_s18@vistec.ac.th
}

\author{
Rattasat Laotaew ${ }^{2}$ \\ rattasatl_pro@vistec.ac.th \\ Thanasan Nilsu ${ }^{1}$ \\ thanasan.n@kvis.ac.th \\ (1) Thanyada Rungrotmongkol ${ }^{3,4, *}$ \\ thanyada.r@chula.ac.th
}

\section{DSarana Nutanong ${ }^{2, *}$ \\ snutanon@vistec.ac.th}

${ }^{1}$ Kamnoetvidya Science Academy (KVIS), Rayong 21210, Thailand

2 School of Information Science and Technology, Vidyasirimedhi Institute of Science and Technology (VISTEC), Rayong 21210, Thailand

3 Program in Bioinformatics and Computational Biology, Graduate School, Chulalongkorn University, Bangkok 10330, Thailand

${ }^{4}$ Biocatalyst and Environmental Biotechnology Research Unit, Department of Biochemistry, Faculty of Science, Chulalongkorn University, Bangkok 10330, Thailand

$\dagger$ These authors contributed equally to this work.

* Corresponding author 
1 Motivation: Lung cancer is a chronic non-communicable disease and is the cancer with the world's

2 highest incidence in the $21^{\text {st }}$ century. One of the leading mechanisms underlying the development of

3 lung cancer in nonsmokers is an amplification of the epidermal growth factor receptor (EGFR) gene.

4 However, laboratories employing conventional processes of drug discovery and development for

5 such targets encounter several pain-points that are cost- and time-consuming. Moreover, high failure

6 rates are caused by efficacy and safety problems during research and development. Therefore, it is

7 imperative to develop improved methods for drug discovery. Herein, we developed a deep learning

8 model with spatial graph embedding and molecular descriptors based on predicting $\mathrm{pIC}_{50}$ potency

9 estimates of small molecules and classifying hit compounds against the human epidermal growth

10 factor receptor (LigEGFR). The model was generated with a large-scale cell line-based dataset

11 containing broad lists of chemical features.

12 Results: LigEGFR outperformed baseline machine learning models for predicting $\mathrm{pIC}_{50}$. Our model

13 was notable for higher performance in hit compound classification, compared to molecular docking

14 and machine learning approaches. The proposed predictive model provides a powerful strategy

15 that potentially helps researchers overcome major challenges in drug discovery and development

16 processes, leading to a reduction of failure to discover novel hit compounds.

17 Availability: We provide an online prediction platform and the source code that are freely available

18 at https://ligegfr.vistec.ist, and https://github.com/scads-biochem/LigEGFR, respectively.

19 Keywords Deep learning · Machine learning · Graph embedding · Drug discovery · QSAR .

20 Epidermal growth factor receptor 


\section{Key points}

- LigEGFR is a regression model for predicting $\mathrm{pIC}_{50}$ that was developed for the human EGFR target. It can also be applied to hit compound classification $\left(\mathrm{pIC}_{50} \geq 6\right)$ and has a higher performance than baseline machine learning algorithms and molecular docking approaches.

- Our spatial graph embedding and molecular descriptors based approach notably exhibited a high performance in predicting $\mathrm{pIC}_{50}$ of small molecules against human EGFR.

- Non-hashed and hashed molecular descriptors were revealed to have the highest predictive performance by using in a convolutional layers and a fully connected layers, respectively.

- Our model used a large-scale and non-redundant dataset to enhance the diversity of the small molecules. The model showed robustness and reliability, which was evaluated by y- randomization and applicability domain analysis (ADAN), respectively.

- We developed a user-friendly online platform to predict $\mathrm{pIC}_{50}$ of small molecules and classify the hit compounds for the drug discovery process of the EGFR target.

Puri Virakarin is a high school student at Kamnoetvidya Science Academy (KVIS). His research interests include Machine Learning, Deep Learning, and Computational Biology.

Natthakan Saengnil is a high school student at Kamnoetvidya Science Academy (KVIS). His research interests include Machine Learning, Deep Learning, and Applied Physics.

Bundit Boonyarit is a Ph.D. student in Information Science and Technology at Vidyasirimedhi Institute of Science and Technology (VISTEC). His research interests include Molecular Machine Learning, Computer-aided Drug Design \& Discovery, and Computational Biology \& Chemistry.

Jiramet Kinchagawat is a visiting researcher at the School of Information Science and Technology, Vidyasirimedhi Institute of Science and Technology (VISTEC). His research interests include Machine Learning, Deep Learning, Drug Discovery, Biological Data Engineering, and Biomedical Engineering.

Rattasat Laotaew is a developer at the School of Information Science and Technology, Vidyasirimedhi Institute of Science and Technology (VISTEC). He specializes in back-end web service, web application, and server management.

Treephop Saeteng is a developer at the School of Information Science and Technology, Vidyasirimedhi Institute of Science and Technology (VISTEC). He specializes in front-end web service and web applications.

Thanasan Nilsu is a Biology and Environmental Science teacher at Kamnoetvidya Science Academy (KVIS). His research interests include Medicinal Chemistry, Organic Chemistry, Phytochemistry, and Biomedical Sciences.

Naravut Suvannang is a postdoctoral researcher at the School of Information Science and Technology, Vidyasirimedhi Institute of Science and Technology (VISTEC). His research interests include Machine Learning, Computer-aided Drug Design \& Discovery, and Bioinformatics.

Thanyada Rungrotmongkol is an Assistant Professor at the Department of Biochemistry, Faculty of Science, Chulalongkorn University. She leads the BioSIM lab. Her research interests include Computer-aided Drug Design \& Discovery, Computational Chemistry, and Bioinformatics.

Sarana Nutanong is an Associate Professor at the School of Information Science and Technology, Vidyasirimedhi Institute of Science and Technology (VISTEC). He leads the Scalable Data Systems lab. His research interests include Machine Learning, Deep Learning, Big Data Analytics, Data Systems, Natural Language Processing, and Computational Biology \& Chemistry. 


\section{Introduction}

Non-communicable diseases (NCDs) are increasingly leading causes of death. Lung cancer is a global health burden and has been reported as the most frequent cancer worldwide; in 2018, lung cancer was the most commonly diagnosed cancer (11.6\% of the total cases) and the leading cause of cancer death (18.4\% of the total cancer deaths) [1]. The leading causes of lung cancer in nonsmokers and those who are not in close contact with smokers are the amplification and hyperactivation of the epidermal growth factor receptor (EGFR). EGFR is a member of receptor tyrosine kinases (RTKs) family located on the cell membrane and the elevated activity of this enzyme results in increased cascades of the RAS-RAF-MEK-ERK and PI3K-AKT-mTOR signaling pathways [2]. Chemotherapy is the traditional treatment, with drugs taken at high doses, but it can cause a number of unpleasant side effects such as dizziness, alopecia, oral and pharyngeal tissue damage, and fatigue [3]. Nowadays, targeted therapy is a new approach to treatment that is developed specifically for cancer targets and has generated agents such as Gefitinib, Erlotinib, Lapatinib, Afatinib, Dacomitinib, Neratinib, Osimertinib, and Brigatinib [4].

Computer-aided drug discovery can help reduce the time and cost in the early stages of drug discovery and development process [5]. The virtual screening technique is a method for accelerating drug discovery and is commonly performed by molecular docking, pharmacophore modeling and mapping, and molecular similarity calculations. However, these traditional techniques have

several disadvantages, such as the inability to identify whether the compound is an inhibitor or an activator for the target. The ligand with the highest score from molecular docking does not automatically have the highest potential to be a useful lead compound [6]. Over the past decade of drug discovery, the use of big data sets, such as bioactivity and drug-target interactions, has been dramatically increased through the development of high-throughput screening (HTS) technologies $[7,8,9]$. Consequently, machine learning and deep learning techniques now play important roles in the pharmaceutical industry by enhancing efficiency in hit compound discovery with a result in decreasing the proportion of drug failures in clinical trials. 
61 Machine learning is a widely used tool that opens vast opportunities in the drug development

62 process. It can be applied to constructing QSAR models, built through various state-of-the-art

63 techniques. QSAR explores the structure of the molecule to predict the activities, such as bioactivity

$64\left(\mathrm{IC}_{50}, \mathrm{EC}_{50}, \mathrm{~K}_{\mathrm{i}}\right.$, and $\mathrm{K}_{\mathrm{d}}$ ), adsorption, distribution, metabolism, elimination, and toxicity (ADMET)

65 properties; it is an essential indicator in selecting lead compounds before they are tested in vitro and

66 in vivo. Generally, QSAR studies in drug discovery are focused on the physicochemical properties

67 of the molecules that trigger similar biological effects [10]. There are prominent published works

68 that used deep learning for predicting molecular properties such as binding affinity [11, 12, 13],

69 drug-target interaction [14], and bioactivity [15]. It can be seen that bioactivity prediction using

70 machine learning methods is helpful in accelerating the drug discovery process. For EGFR, there

71 have been various machine learning models developed to predict $\mathrm{IC}_{50}$ of ligands against wild type

72 EGFR that were based on small scale datasets (up to 290 compounds) [16, 17, 18, 19, 20, 21, 22].

73 But machine learning models that are based on small-scale compound datasets may not accurately

74 predict properties owing to out-of-feature domains and low diversity of substructure features in the

75 dataset molecules.

76 To tackle the problems and pitfalls in the previous methods of drug discovery, we first developed a

77 machine learning model to predict $\mathrm{pIC}_{50}$ of small molecules using the human EGFR as the target

78 and termed it LigEGFR. The development of this model using a large-scale cell line-based and

79 non-redundant dataset is described here. The convolution spatial graph embedding network (C-

80 SGEN) [23] [24] together with the deep neural network (DNN) technique was modified and applied

81 for model training in this work. C-SGEN is comprised of convolution spatial graph embedding

82 layers (C-SGELs) which are constructed by graph convolutional networks. C-SGEL is introduced

83 to maintain the spatial relationship of atoms in a molecule by using the GraphCov features [25].

84 The molecular graph represented by an adjacency matrix and a node matrix is used as an input of

85 C-SGELs.

86 The main contributions of our work are as follows:

87 (i) We employed the C-SGEN algorithm with architecture adaption and $\mathrm{DNN}$ for $\mathrm{pIC}_{50}$ prediction with a large-scale and non-redundant dataset of bioactive compounds against human EGFR. 
89 (ii) The non-hashed and hashed molecular fingerprints were considered for convolutional (Conv) layers and fully connected (FC) layers, respectively.

91(iii) We constructed a user-friendly web service with compatibility for all devices, 92 https://ligegfr.vistec.ist, and Python executable script, https://github.com/scads93 biochem/LigEGFR, for predicting $\mathrm{pIC}_{50}$ and classifying hit compounds of small molecules 94 against human EGFR.

\section{Methods}

96 The overview of LigEGFR is shown in Figure 1. We used the large cell line-based and non-redundant

97 dataset to retrieve broad features of small molecules. These features were applied to LigEGFR, 98 comprising C-SGELs for the GraphCov features, FC layers, and Conv layers for different fingerprint 99 combinations. LigEGFR was then compared to the baseline machine learning algorithms: C-SGEN, $100 \mathrm{CNN}$, and random forest (RF). Also, the classification performance of the conventional virtual 101 screening techniques such as molecular docking (CCDC GOLD and AutoDock Vina programs), was 102 determined. The model assessment was performed by the y-scrambling technique and applicability 103 domain analysis (ADAN). The details of the methodologies are particularized in the following 104 subsections. For web service construction, the description is provided in the supplemental section $105 \mathrm{~S} 1^{\dagger}$.

\subsection{Data preparation}

107 The 37,753 substances (90,118 data points) known to inhibit the activity of human EGFR were

108 collected from the Reaxys database [26]. The crucial information consisted of the ligand structures

109 in the format of SMILES (Simplified Molecular-Input Line-Entry System) and the pIC $_{50}$ values

110 derived as $-\log \left(\mathrm{IC}_{50}\right)$. The data from wild type human EGFR and in vitro experiment as well

111 as from cell-based assay were selected. The redundant compounds with the same SMILES and

112 those with missing pIC $_{50}$ values or SMILES were removed; salt derivatives in SMILES was also

113 eliminated. After that, we removed outliers by considering the rule of five (RO5) criterion, with (i)

114 hydrogen bond donors, (ii) hydrogen bond acceptors, (iii) molecular weight, and (iv) the octanol- 


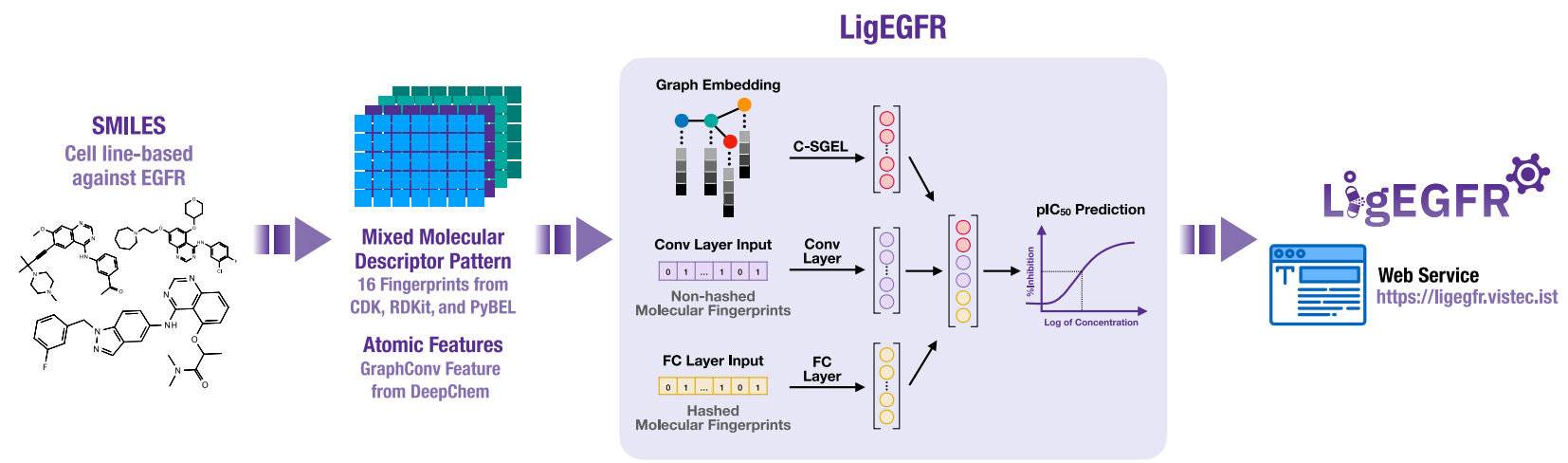

Figure 1: This overview represents LigEGFR construction. The dataset, SMILES strings, is converted into the GraphCov features, non-hashed molecular fingerprints, and hashed molecular fingerprints. LigEGFR encodes these features to construct a predictive model by using C-SGEL for the GraphCov features, the Conv layers for the non-hashed molecular descriptors, and the FC layers for the hashed molecular descriptors.

115 water partition coefficient $(\log \mathrm{P})$. We used the interquartile range method (IQR) by

$$
I Q R=Q_{3}-Q_{1}
$$

116 and outliers were qualified for exclusion if they fell outside the range as follows:

$$
\left[Q_{1}-(k \times I Q R), Q_{3}+(k \times I Q R)\right]
$$

117 where $k=3$. In this process, Pandas [27], NumPy [28], Scipy [29], Matplotlib [30], scikit-learn 118 [31], RDKit [32], PyBEL [33], and Seaborn [34] packages were used for data management and 119 processing.

\section{$120 \quad 2.2$ Molecular fingerprints}

121 The SMILES of compounds were encoded into a molecular fingerprint comprising a set of molecular

122 descriptors or features. In this work, we made 16 molecular fingerprints from the CDK [35], RDKit

123 [32], and PyBEL [33] packages (see supplemental Table $\mathrm{S} 1^{\dagger}$ ). These descriptors were represented

124 in Boolean form. The types of molecular fingerprints were divided into two groups, non-hashed and hashed molecular fingerprints. Furthermore, the fingerprints were reduced in dimension with 
126 different correlation coefficients in feature selection and the number of principal components in

127 the PCA method. For the feature selection method, the variance threshold was fixed to 0.001,

128 and the correlation coefficient was varied from 0.75 to 0.90 . For the PCA method, the number of

129 components was altered from 50 to 800.

\section{$130 \quad 2.3 \quad$ Dataset splitting}

131 Our dataset was divided into training set and test set using the Train/Test split method [31]. The

132 training set ratio to the test set was $80: 20$, where $80 \%$ of the final dataset was used as an internal set

133 (2,794 compounds) and the remaining $20 \%$ used for the test set were unseen data (699 compounds).

134 The internal set was then separated into a training set (72\%, 2,515 compounds) and validation set

135 (8\%, 279 compounds).

\section{$136 \quad 2.4$ Model development}

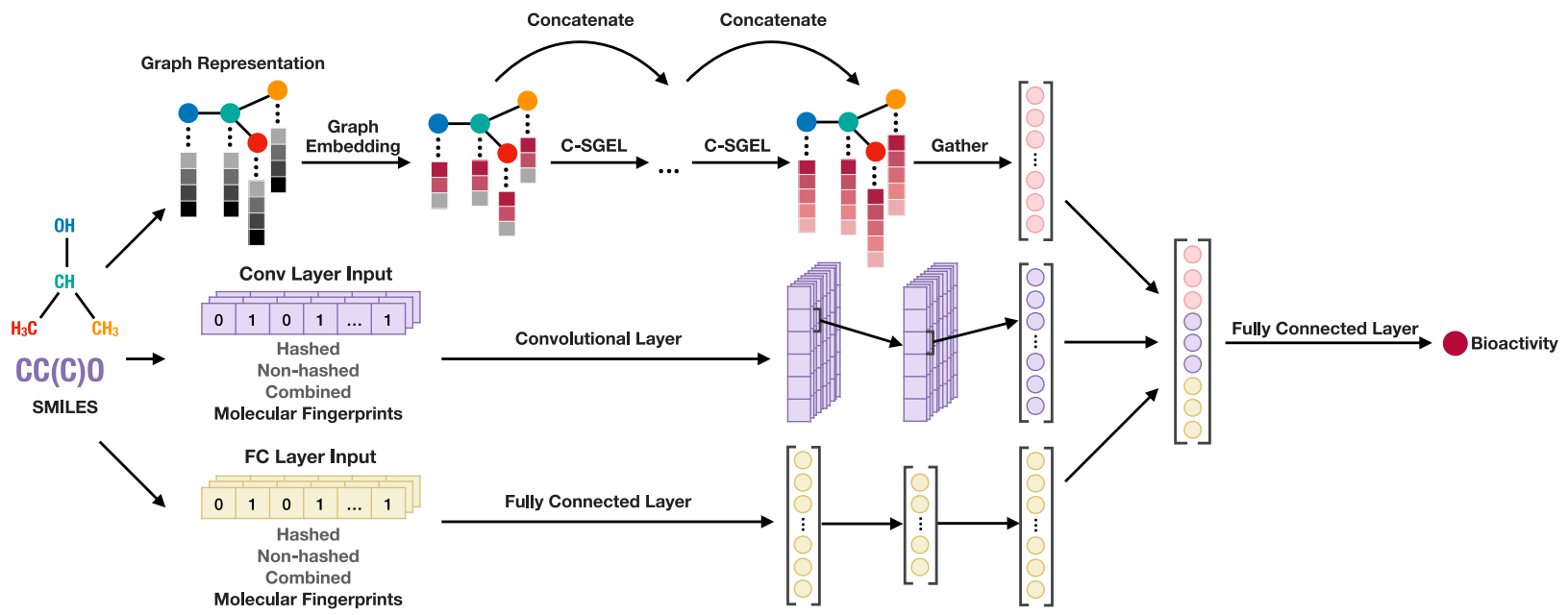

Figure 2: Diagram of the LigEGFR architecture contains the C-SGEN algorithm with architecture adaptation for graph embedding and the DNN algorithm for molecular fingerprints.

137 Figure 2 shows the structure of a LigEGFR architecture. The C-SGEN algorithm [23] was applied

138 for LigEGFR construction with a modification in the architecture. Normally, each molecule is

139 represented by a molecular graph and hashed and non-hashed molecular descriptors. The connection

140 between atoms is constructed as a graph that is initialized from the node matrix $(X)$ and an adjacency

141 matrix $(A)$. By regarding the atomic connection between atoms $i$ and $j$, if it appears then $A_{i, j}=1$; 
142 otherwise, $A_{i, j}=0$. The initial node matrix $(X)$ is proposed by a set of atoms $x_{i}$ :

$$
X=\left\{x_{1}, x_{2}, \ldots, x_{n}\right\}
$$

143 where each atom $x_{i}$ is an $m$-dimensional vector. The vector is extracted from DeepChem [25]

144 and encoded into a one-hot vector by the atomic features shown in supplemental Table S2 ${ }^{\dagger}$. The

145 propagation function used in C-SGEN follows the equation

$$
X^{l}=\operatorname{Concat}\left(\sigma\left(D^{-1 / 2} \hat{A} D^{-1 / 2} X^{l-1} W^{l}+b^{l}\right), X^{l}\right)
$$

146 where $\hat{A}=A+I_{n}$ and $D$ is the diagonal matrix containing degree of each node in $\hat{A} . D^{-1 / 2} \hat{A} D^{-1 / 2}$

147 is a symmetric normalization of an adjacency matrix, where the propagation of each adjacent node

$148(i, j)$ is normalized by the degree of both nodes $i$ and $j[36] . W^{l} \in \mathbb{R}^{d_{l} \times d_{l}^{n n}}$ and $b \in \mathbb{R}^{n \times d_{l}^{n n}}$

149 represent the learnable weight and bias, respectively, where $d_{l-1}$ is the dimension of layer $l-1$

150 and $d_{l}^{n n}$ is the dimension resulted from the neural network computation in layer $l$. The feature

151 vector resulted from the neural network is a matrix with dimension $d_{l}^{n n} \times n$ concatenated to the

152 original feature vector $X^{l-1}$ resulted in the output matrix $X^{l}$ with dimension $n \times\left(d_{l-1}+d_{l}^{n n}\right)$. The

153 illustration of C-SGEN propagation is shown in Figure 3.

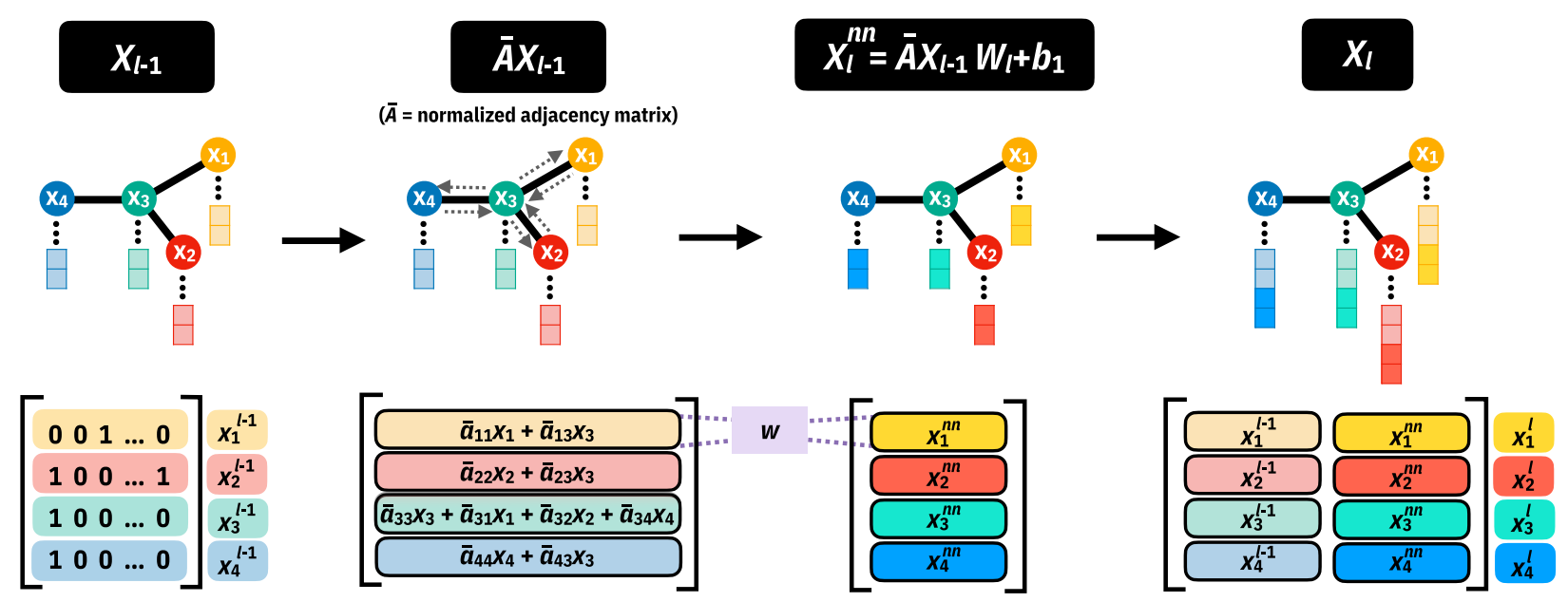

Figure 3: This schematic of C-SGEN propagation contains a visualization of the step in graph feature propagation that includes the multiplication of the feature with the normalized adjacency matrix $\bar{A}$ which is equal to $D^{-1 / 2} \hat{A} D^{-1 / 2}$. Afterward, the hidden learnable computation layer of weight and bias is used to extract the feature and concatenate back to the previous layer. 
154 After C-SGEN, the feature matrix $X^{f}$ comprises each atom feature vector $x_{i}^{f}$, where $f$ is the final

155 layer of C-SGEN. In order to obtain an order-invariant feature for one molecule, the molecular 156 graph representation $x_{\text {graph }}$ is calculated by a summation of all atomic features $x_{i}^{f}$ as follows.

$$
x_{\text {graph }}=\sum_{i=1}^{n} x_{i}^{f}
$$

157 Here the dimension of molecular graph representation $x_{\text {graph }}$ is the same as the atomic feature

158 vector $x_{i}^{f}$. Afterward, the molecular fingerprint is used as another input of the model to confirm

159 the feature's generalization. These molecular fingerprints can be split into hashed and non-hashed

160 fingerprints for the input of the Conv layers and FC layers. The fingerprints of each molecule can be

161 expressed as $x^{0}$, and the output from the $l$ th hidden layer can be represented as $x^{l}$ using the equation.

162

$$
x^{l}=\sigma\left(W^{l} x^{l-1}+b^{l}\right)
$$

163 where $\sigma, W^{l}$ and $b^{l}$ are activation function, learnable weight matrix, and bias for the $l$ th hidden

164 layers, respectively. Then, the output from the last layer of both Conv and FC layers are concatenated

165 together into an updated molecular fingerprint, denoted by $x_{\text {finger }}$.

166 Lastly, $x_{\text {graph }}$ and $x_{\text {finger }}$ are concatenated into the final molecular representation, $x_{m o l}$. This $x_{m o l}$

167 is used to predict the $\mathrm{pIC}_{50}\left(y_{\text {predicted }}\right)$ of the molecule through the FC layers as follows.

$$
x_{\text {mol }}=\left[x_{\text {graph }}, x_{\text {finger }}\right]
$$

168

$$
y_{\text {predicted }}=W x_{m o l}+b
$$

169 where $W$ is the weight matrix and $b$ is the bias.

170 To find the fingerprint pattern that is followed through with a high-performance model, we have

171 changed the combination of fingerprints used as input to the Conv layers and FC layers as shown

172 below:

173 1. The hashed and non-hashed fingerprints were applied in the Conv layers and FC layers, respectively. 
175 2. The hashed and non-hashed fingerprints were used in the FC layer and Conv layers, respectively.

176 3. Both hashed and non-hashed fingerprints (all fingerprints) were applied in both FC and Conv 177 layers.

178 In addition, hyperparameter tuning was applied to this architecture by adjusting BatchSize, $l r$,

179 ch_num, $k$, and csgellayer. The detail of hyperparameter tuning is described in supplemental section $180 \mathrm{~S}^{\dagger}$.

\section{$181 \quad 2.5 \quad$ Model assessment}

\section{$182 \quad$ 2.5.1 Predictive performance}

183 We evaluated the performance of the regression models by using the coefficient of determination

$184\left(R^{2}\right)$ and root mean square error $(R M S E)$.

185 The $R^{2}$ indicates how well the predicted values compared with the observed values

$$
R^{2}=1-\frac{\sum_{i=1}^{N}\left(y_{i}^{r e a l}-y_{i}^{\text {pred }}\right)^{2}}{\sum_{i=1}^{N}\left(y_{i}^{r e a l}-\bar{y}^{r e a l}\right)^{2}}
$$

186 while the RMSE represents the root mean square or quadratic mean of the difference between the

187 predicted value and the observed value.

$$
R M S E=\sqrt{\sum_{i=1}^{N} \frac{\left(y_{i}^{\text {real }}-y_{i}^{\text {pred }}\right)^{2}}{N}}
$$

188 where $y^{\text {real }}$ is the observed $\mathrm{pIC}_{50}$ value from experiments, $y^{\text {pred }}$ is the predicted $\mathrm{pIC}_{50}$ value from

189 the machine learning model, and $N$ is the number of compounds used for the performance test.

190 Each metric was used to evaluate the training set $\left(R_{\text {train }}^{2}\right.$ and $\left.R M S E_{\text {train }}\right)$, validation set $\left(Q_{v a l}^{2}\right.$ and

$\left.191 R M S E_{v a l}\right)$ and test set $\left(Q_{\text {test }}^{2}\right.$ and $\left.R M S E_{\text {test }}\right)$ by averaging statistical values and changing the random 192 seed. 


\subsubsection{Y-scrambling validation}

194 Basically, the robustness of the model is evaluated by the y-scrambling or y-randomization technique.

195 This procedure can predict the performance of the model by comparing the observed values $\left(y^{\text {real }}\right)$

196 to the model built from shuffled $Y$ - leaving the entire $X[37,38]$. If the original descriptors cannot

197 predict the random $\mathrm{pIC}_{50}$ value which is calculated from the shuffled model, the original model is

198 robust. The basic leave-one-out (LOO) technique was applied for statistics of the scrambled models.

199 In this experiment, we employed the $100 \mathrm{y}$-scrambling models for comparing $Q_{\text {test }}^{2}$ to the initial 200 model.

\section{2.5.3 Applicability domain analysis (ADAN)}

202 The applicability domain (AD) is the chemical space, structure, or knowledge from a training

203 set in which the model makes reliable predictions and is considered to be applicable [39]. The

204 AD determines the extent of the data for which the predictions of the model are valid. In other

205 words, the prediction must be an interpolation. Typically, the AD is calculated by a range-based

206 method, geometric method, distance-based method, or probability density distribution-based method

207 [40]. We used k-nearest neighbors ( $\mathrm{kNN}$ )-based and PCA bounding box methods for evaluation.

208 These methods are simple to understand and interpret. In the kNN-based method, if the Euclidean

209 distances between a query and the training set exceed the ADT, the prediction is not reliable, and

210 vice versa. ADT was calculated as follows for new substance prediction [41].

$$
\mathrm{ADT}=\bar{y}+Z \sigma
$$

211 Here, $\bar{y}$ is an average of the Euclidean distances of $\mathrm{kNN}$ in the training set, in which $k=50$ as the

212 square root of the training set number [42], $Z$ is an arbitrary parameter ( 0.5 by default), and $\sigma$ is a

213 standard deviation of the Euclidean distances.

214 For the PCA bounding box, a 3D principal component (PC) space was generated for the training set, 215 test set, approved drug set, and clinical trial compound set. Each point in the PC space represents a 
216 compound, so the training set's chemical space can be found. If a query is in the bounding box, the

217 prediction is reliable (interpolation) [43].

\section{$218 \quad 2.6$ Baseline comparison}

219 We have compared the efficiency of our LigEGFR with baseline machine learning algorithms and

220 the conventional molecular docking methods in predicting $\mathrm{pIC}_{50}$ value and whether a query is a

221 hit compound $\left(\mathrm{pIC}_{50} \geq 6\right)[44]$. The receiver operating characteristic (ROC) curves, plotting the

222 true-positive rate against the false-positive rate, were obtained from both approaches by changing the

223 threshold used to classify each ligand [45]. Moreover, the enrichment plot, plotting the percentage

224 of identified hit compounds against the selected top compounds, indicates how rich the correctly

225 predicted hit compound is in the whole ranked dataset. The area under the ROC curve (AUC),

226 and the enrichment plot were used as metrics for performance comparison. Furthermore, the

227 balanced-accuracy, precision, recall, F1-score, and Matthews correlation coefficient (MCC) were

228 also evaluated to determine the efficiency of the classification task. The definitions of these metrics

229 are shown below.

$$
\text { Balanced Accuracy }=\left[\frac{T P}{T P+F N}+\frac{T N}{T N+F P}\right] / 2
$$

230

$$
\text { Precision }=\frac{T P}{T P+F P}
$$

231

$$
\text { Recall }=\frac{T P}{T P+F N}
$$

232

$$
\text { F1-score }=\frac{2 \times \text { Precision } \times \text { Recall }}{\text { Precision }+ \text { Recall }}
$$

233

$$
\mathrm{MCC}=\frac{T P \times T N-F P \times F N}{\sqrt{(T P+F P)(T P+F N)(T N+F P)(T N+F N)}}
$$

$234 T P$ (true-positives) and $F N$ (false negatives) denote the numbers of known active compounds 235 predicted to be active compounds $\left(\mathrm{pIC}_{50} \geq 6\right)$ and inactive compounds $\left(\mathrm{pIC}_{50}<6\right)$. $T N$ (true 
236 negatives) and FP (false positives) indicate the numbers of known inactive compounds predicted to

237 be inactive compounds and active compounds.

\section{2.6.1 Machine learning-based approaches}

239 The baseline evaluation was determined from machine learning techniques by RF, CNN, and

240 C-SGEN algorithms. We developed the RF model by using the recursive feature elimination

241 (RFE) technique with Adjusted $R^{2}$. The CNN model was applied using Conv and FC layers with

242 reduced hashed and non-hashed fingerprints. The C-SGEN algorithm was performed as a controlled

243 experiment.

244 Additionally, RF and CNN models were tuned by hyperparameter optimization. The detailed 245 methods are described in supplemental section $\mathrm{S}^{\dagger}, \mathrm{S}^{\dagger}$, and $\mathrm{S}^{\dagger}$ for RF, CNN, and C-SGEN 246 algorithms, respectively.

\section{2.6.2 Molecular docking-based approaches}

248 We applied the molecular docking to calculate fitness scores between a list of test set ligands and

249 EGFR protein. Generally, the ligand with the lowest binding free energy $\left(\Delta \mathrm{G}_{\text {bind }}\right)$ which has the

250 highest binding affinity to the drug target has the greatest chance to be an efficient hit compound.

251 Each ligand's fitness score from the test set was obtained from the EGFR tyrosine kinase in both

252 active and inactive conformations: PDB entry codes 1M17 [46] and 1XKK [47]. These protein

253 structures were prepared using the python script, clean_pdb_keep_ligand.py through Rosetta3 [48].

254 All ligand geometries in the test set were optimized by the MMFF94 force field with RDKit and

255 subsequently converted into the .mol2 file. The CCDC GOLD (the Genetic Optimisation for Ligand

256 Docking) version 5.8.0 [49] with ChemPLP (Piecewise Linear Potential) fitness score [50], and

257 AutoDock Vina 1.1.2 [51] were implemented for molecular docking. The described details of molecular docking are provided in supplemental section $\mathrm{S}^{\dagger}$ and $\mathrm{S}^{\dagger}$. 


\section{Results and discussion}

\subsection{Predictive performance for regression tasks}

Table 1: LigEGFR, C-SGEN, CNN, and RF models' predictive performance is indicated for regression tasks. The results of all models were computed and tuned with the various hyperparameters, with the exception of C-SGEN which was trained on the original hyperparameter.

\begin{tabular}{lccccccc}
\hline \multirow{2}{*}{ Algorithm } & \multicolumn{3}{c}{ Training set } & \multicolumn{2}{c}{ Validation set } & \multicolumn{2}{c}{ Test set } \\
\cline { 2 - 7 } & $R_{\text {train }}^{2}$ & $R M S E$ & $Q_{\text {valid }}^{2}$ & $R M S E$ & $Q_{\text {test }}^{2}$ & $R M S E$ \\
\hline LigEGFR & 0.986 & 0.175 & 0.642 & 0.903 & $\mathbf{0 . 7 0 2}$ & $\mathbf{0 . 8 0 2}$ \\
C-SGEN & 0.972 & 0.246 & 0.605 & 0.943 & 0.661 & 0.858 \\
CNN & 0.885 & 0.499 & 0.633 & 0.910 & 0.692 & 0.817 \\
RF & 0.958 & 0.306 & 0.672 & 0.851 & 0.683 & 0.828 \\
\hline
\end{tabular}

261 As shown in Table 1, the LigEGFR model, after tuning the hyperparameter with the feature selection

262 technique, outperformed all baseline algorithms for the regression task $\left(Q_{\text {test }}^{2}=0.702\right.$ and $R M S E$ $263=0.802$ ). The best hyperparameters for LigEGFR were BatchSize $=32, l r=5 \times 10^{-5}$, ch_num $=$ $2644, k=8$, and csgellayer $=4$. More importantly, LigEGFR also provided a more accurate $\mathrm{pIC}_{50}$ 265 prediction for the test set compared to those of the baseline algorithms (see Figure 4). The predictive 266 performance of the test set described by $Q_{\text {test }}^{2}$ in QSAR task, should be $\geq 0.6$ [52]. LigEGFR

267 composes graph embedding layers that are gathered from an atomic feature representation of the 268 molecule, which is based on the C-SGEN algorithm [23]. The advantage of the graph-based method 269 for molecular representation is that it is able to pull out essential features of molecular information 270 including the neighboring atoms.

271 The additive non-hashed and hashed molecular descriptors embedded on Conv and FC layers,

272 have improved the predictive performance and generalized model. The feature analysis of these

273 molecular descriptors is described in supplemental section $\mathrm{S}^{\dagger}{ }^{\dagger}$. From Table 2, we noticed that

274 non-hashed molecular descriptors on the Conv layers outperformed other arrangements in predictive

275 performance. The non-hashed molecular descriptor is a feature that represents the presence of the 276 substructures of a molecule such as atom type, neighboring atoms, atomic groups, bonds, etc. The

277 Conv layers with substructure molecular fingerprints may learn relationships and similar features 278 of the various small molecules to attain a global representation of the information that influences 


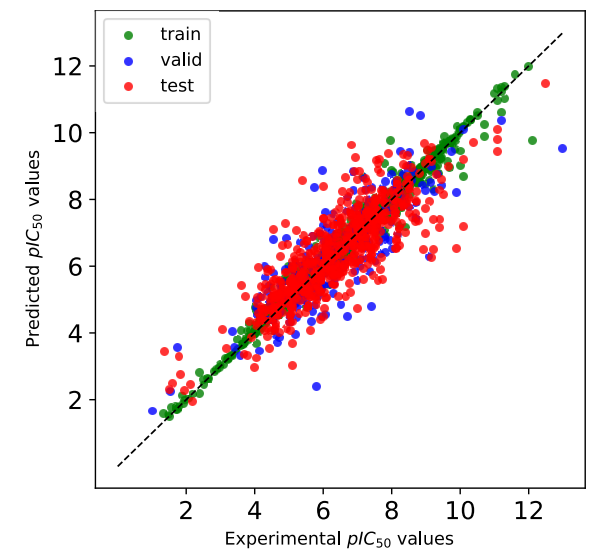

(a) LigEGFR

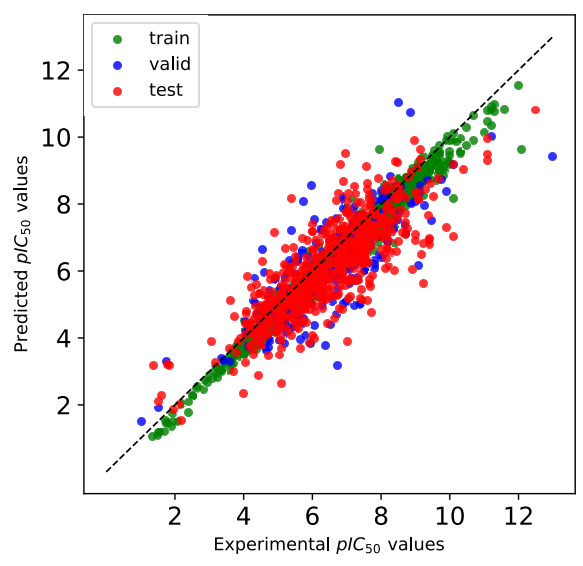

(c) $\mathrm{CNN}$

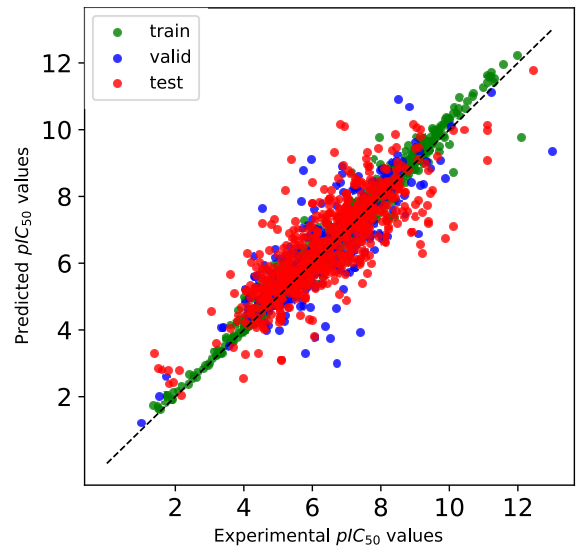

(b) C-SGEN

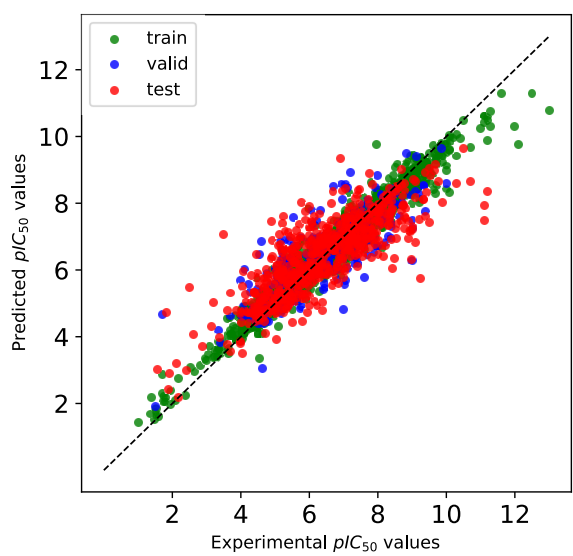

(d) RF

Figure 4: Comparison of relationships between predicted and experimental $\mathrm{pIC}_{50}$ values generated by for (a) LigEGFR, (b) C-SGEN, (c) CNN, and (d) RF. The dashed line shows where the points should ideally lie.

279 the physicochemical properties of the molecule. The non-hashed molecular fingerprints on the

280 Conv layers and the hashed molecular fingerprints on the FC layers with cut-off values by feature

281 selection, 0.001 for low variance and 0.90 for collinearity, exhibit the highest predictive performance

$282\left(Q_{\text {test }}^{2}=0.696\right.$ and $\left.R M S E_{\text {test }}=0.813\right)$ without hyperparameter tuning.

283 In comparing the C-SGEN algorithm with the original hyperparameter, this algorithm has influences

284 that decrease the predictive performance because the dataset did not conform to the original

285 hyperparameter setting. Moreover, the additive non-hashed and hashed molecular descriptors

286 were not applied to Conv and FC layers, thus it was inadequate for predicting the $\mathrm{pIC}_{50}$ value.

287 The types and shapes of features are prominent requirements for a learning process using proper 
bioRxiv preprint doi: https://doi.org/10.1101/2020.12.24.423424; this version posted December 24, 2020. The copyright holder for this preprint (which was not certified by peer review) is the author/funder, who has granted bioRxiv a license to display the preprint in perpetuity. It is made available under aCC-BY-NC-ND 4.0 International license.

Table 2: The predictive performance based on LigEGFR, trained by Conv and FC layers with a combination of molecular fingerprint patterns. The feature selection technique was completed for molecular fingerprints prior to hyperparameter optimization.

\begin{tabular}{|c|c|c|c|c|c|c|c|c|}
\hline \multirow{2}{*}{\multicolumn{3}{|c|}{ Pattern of molecular fingerprint }} & \multicolumn{6}{|c|}{ Predictive performance } \\
\hline & & & \multicolumn{2}{|c|}{ Training set } & \multicolumn{2}{|c|}{ Validation set } & \multicolumn{2}{|c|}{ Test set } \\
\hline Conv layers & FC layers & Cut-off value & $R_{\text {train }}^{2}$ & $R M S E$ & $Q_{\text {valid }}^{2}$ & $R M S E$ & $Q_{\text {test }}^{2}$ & $R M S E$ \\
\hline Hashed & Non-hashed & {$[0.001,0.75]$} & 0.996 & 0.099 & 0.617 & 0.929 & 0.649 & 0.873 \\
\hline Non-hashed & Hashed & {$[0.001,0.75]$} & 0.994 & 0.117 & 0.642 & 0.899 & 0.693 & 0.816 \\
\hline All & All & {$[0.001,0.75]$} & 0.995 & 0.109 & 0.632 & 0.911 & 0.689 & 0.822 \\
\hline Hashed & Non-hashed & {$[0.001,0.80]$} & 0.996 & 0.099 & 0.623 & 0.922 & 0.656 & 0.865 \\
\hline Non-hashed & Hashed & {$[0.001,0.80]$} & 0.994 & 0.120 & 0.639 & 0.902 & 0.694 & 0.815 \\
\hline All & All & {$[0.001,0.80]$} & 0.995 & 0.108 & 0.628 & 0.916 & 0.691 & 0.818 \\
\hline Hashed & Non-hashed & {$[0.001,0.85]$} & 0.996 & 0.100 & 0.628 & 0.916 & 0.659 & 0.860 \\
\hline Non-hashed & Hashed & {$[0.001,0.85]$} & 0.993 & 0.125 & 0.640 & 0.902 & 0.692 & 0.819 \\
\hline All & All & {$[0.001,0.85]$} & 0.994 & 0.112 & 0.628 & 0.916 & 0.690 & 0.821 \\
\hline Hashed & Non-hashed & {$[0.001,0.90]$} & 0.996 & 0.100 & 0.627 & 0.917 & 0.663 & 0.855 \\
\hline Non-hashed & Hashed & {$[0.001,0.90]$} & 0.993 & 0.125 & 0.636 & 0.906 & 0.696 & 0.813 \\
\hline All & All & {$[0.001,0.90]$} & 0.994 & 0.112 & 0.624 & 0.920 & 0.694 & 0.814 \\
\hline
\end{tabular}

Note: Cut-off values [I,II]; I: low variance of molecular descriptors less than defined value will not be considered. II: collinearity of molecular descriptors more than a defined value will not be considered. can predict $\mathrm{pIC}_{50}$, because this model was optimized with the hyperparameters and embedded with

290 non-hashed and hashed molecular descriptors into different FC and Conv layers. For the RF

291 model, molecular descriptors have been shown to have high performance in predicting pIC $_{50}$, quite

292 equivalent to the $\mathrm{CNN}$ algorithm, because a molecular descriptor as a binary digit has the ability to

293 participate in the ensemble learning process on the decision tree [53]. Consequently, the molecular

294 graph information on C-SGEL plays a significant role in enhancing the predictive performance of

295 the regression model.

296 All results of hyperparameter tuning for LigEGFR, CNN, and RF algorithms are available in 297 supplemental Table $\mathrm{S} 7^{\dagger}, \mathrm{S} 8^{\dagger}$, and $\mathrm{S} 9^{\dagger}$, respectively. 


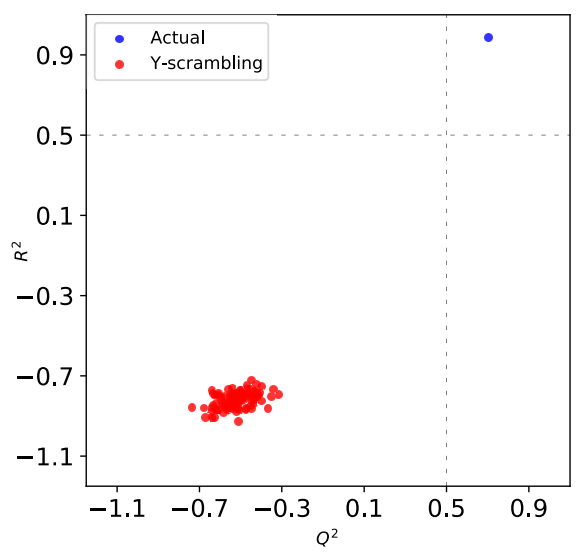

(a) LigEGFR

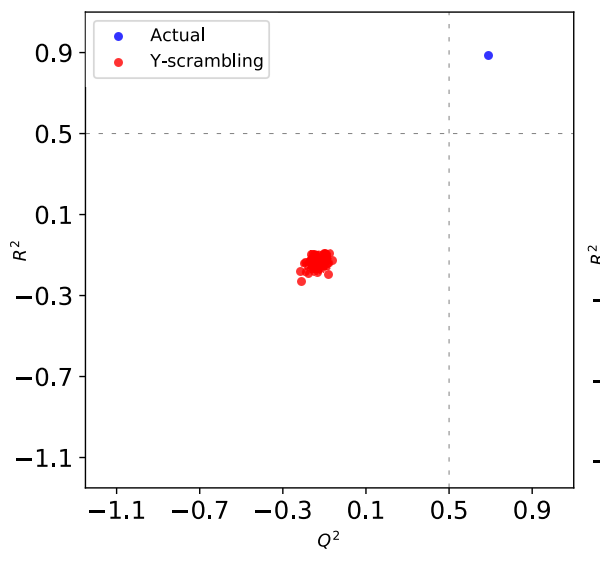

(c) $\mathrm{CNN}$

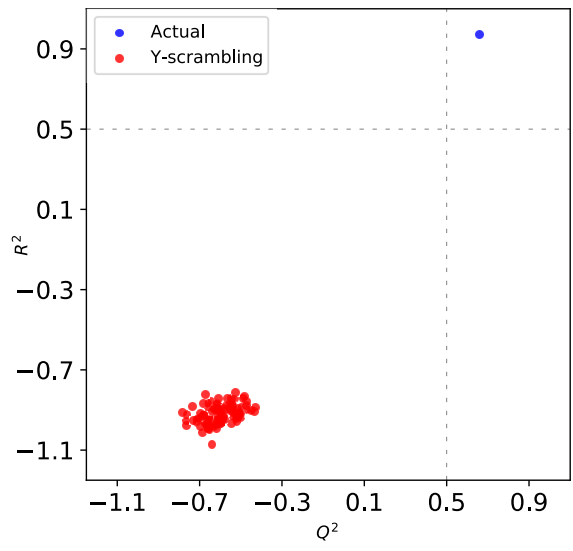

(b) C-SGEN

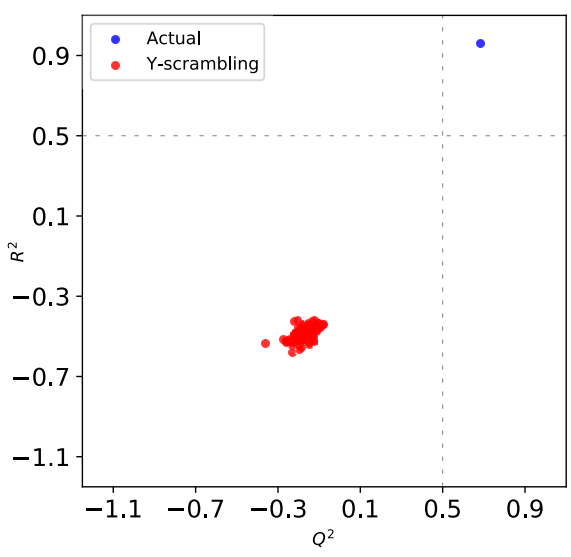

(d) RF

Figure 5: Scatter plots of y-scrambling validation for (a) LigEGFR, (b) C-SGEN, (c) CNN, and (d) RF models.

\subsection{Reliability validation of models}

\subsubsection{Y-scrambling}

300 The y-scrambling is a statistical technique for assessing the robustness of the models. Wold et al.

301 [54] described a criterion to decide an agreed model, based on the $Q_{\text {test(rand) }}^{2}$ and $R_{\text {train(rand) }}^{2}$. If the test

302 provides $Q_{\text {test }(\text { rand })}^{2}$ and $R_{\text {train(rand })}^{2}<0.2$, a result in the model is not a chance correlation to predict a

303 scrambled $Y$ value. Figure 5 shows the plots that the models obtained from actual data (blue dot)

304 arranged in the top right quadrant, thereby suggesting robust models. Meanwhile, the red dots are in

305 the bottom left quadrant $\left(Q_{\text {test (rand) }}^{2}\right.$ and $\left.R_{\text {train(rand })}^{2}<0.2\right)$, indicating the low-performance to predict 
306 shuffled $Y$ values calculated from the original $X$. Hence, our LigEGFR and other models trained

307 on the real dataset have high robustness.

\subsubsection{Loss curves}

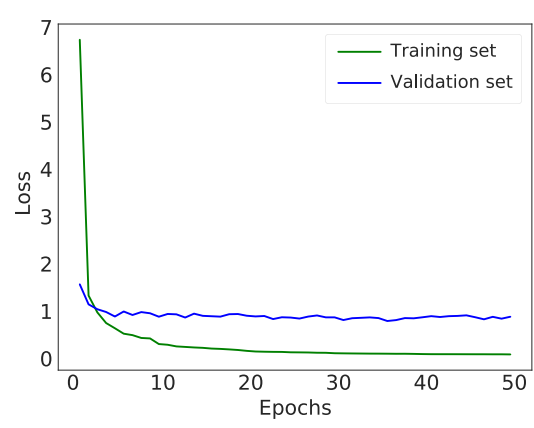

(a) LigEGFR

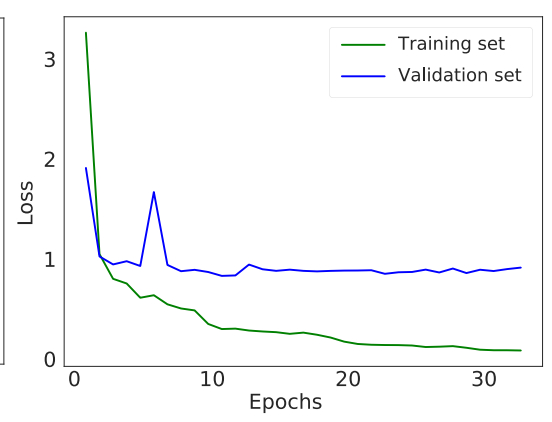

(b) C-SGEN

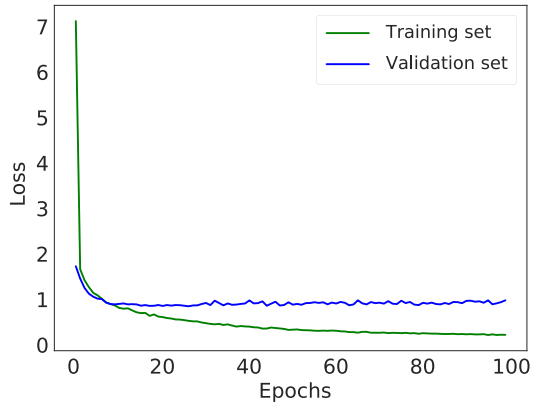

(c) $\mathrm{CNN}$

Figure 6: Loss curves of the training set and validation set are indicated by the green and blue curves, respectively.

Generally, the correctness of the error function of neural networks is indicated by the loss function,

310 which must be minimized. The loss curve emerges to explain model learning and model performance

311 by optimum, overfitting, or underfitting. In this experiment, MSE or $L 2$ was used for the loss function.

312 The MSE is mostly applied for regression problems, estimated by the average of squared differences

313 between the predicted and real values. As shown in Figure 6, the loss curves presented a sharp

314 drop to reach an optimal value in the training set. Meanwhile, the loss curves of the validation set

315 remained constant, a convergence state. We point out that all models were not overfitting, since the

316 loss curves of the validation set and training set were consistent.

\section{3.2.3 Applicability domain analysis (ADAN)}

Table 3: Applicability domain analysis with the kNN-based method for the test set, approved drug set, and clinical trial compound set, where $k=50$

\begin{tabular}{llll}
\hline & Test set & Approved drug set & Clinical trial compound set \\
\hline Number of compounds & 699 & 8 & 18 \\
Reliability & $72.82 \%$ & $87.50 \%$ & $72.22 \%$ \\
\hline
\end{tabular}


318 Evaluating the model's applicability, i.e. assessing whether it is appropriate to the diversity of

319 molecular scaffolds and functional groups, is a crucial step in QSAR method development. We note

320 the results of ADAN by the PCA bounding box and the kNN-based methods, in Figure 7 and Table

3213 , respectively. The $\mathrm{kNN}$-based method provides quantitative data of $\mathrm{AD}$; the PCA bounding box

322 method gives visual and qualitative information of AD. For the kNN-based method, more than $70 \%$

323 of the data in the test set, approved drug set, and clinical trial compound set were in the applicability

324 domain. For the PCA bounding box method, it can be clearly seen that the majority of the data in

325 all sets are in the domain of the training set or interpolation. The results showed that the training

326 set has a large chemical space representing the test set, commercial drugs, and clinical trial drugs.

327 Consequently, our model can reliably predict a new substance as having a high chance to be a lead compound.

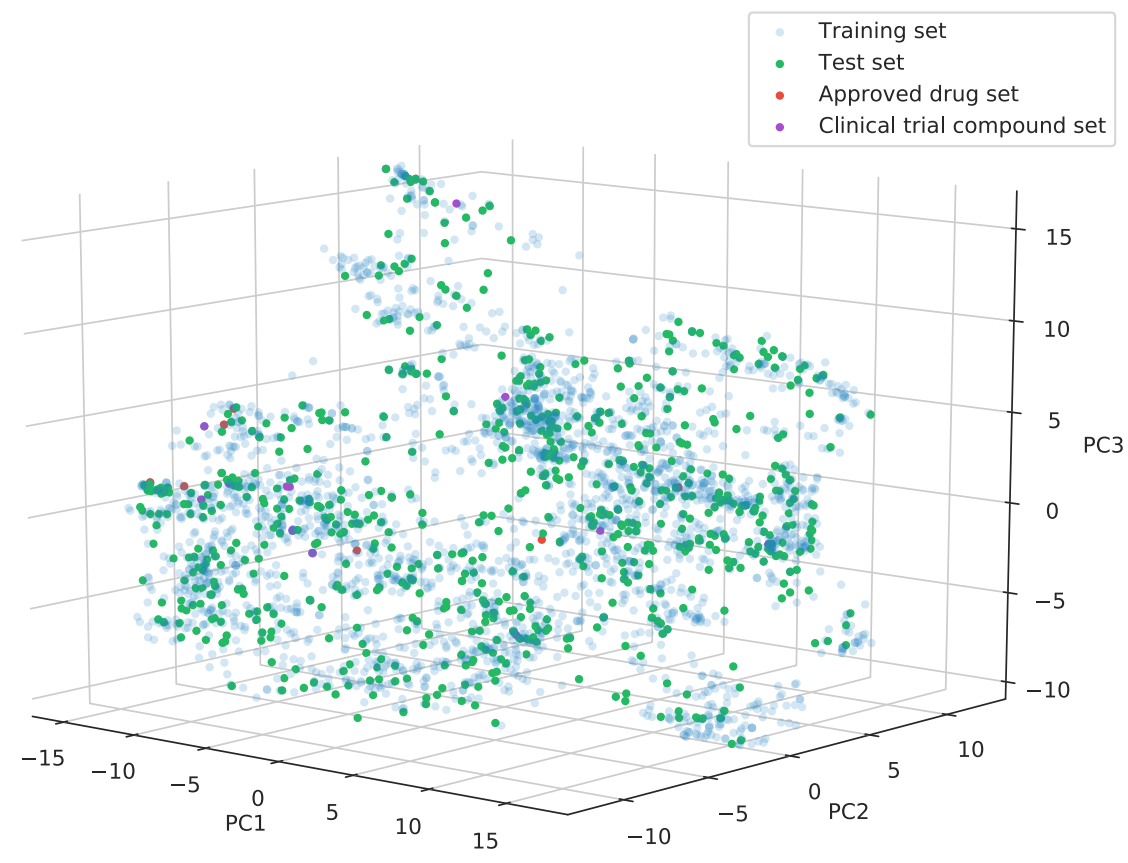

Figure 7: The 3D visualization of the applicability domain shown by the PCA bounding box method.

330 The utility of virtual screening can be compared to molecular docking in the task of classifying 331 active and inactive compounds. Wang et al. [55] and Plewczynski et al. [56] have recognized that 
332 the CCDC GOLD has the best accuracy for the top-scored pose, while Autodock Vina has the best

333 scoring power for both the top-scored and the best pose.

Table 4: Predictive performance for classification tasks attained by LigEGFR, C-SGEN, CNN, and RF models.

\begin{tabular}{llllll}
\hline Algorithm & Balanced Accuracy & Precision & Recall & F1-score & MCC \\
\hline LigEGFR & $\mathbf{0 . 8 6 7}$ & 0.894 & 0.890 & 0.892 & $\mathbf{0 . 7 3 2}$ \\
C-SGEN & 0.863 & 0.883 & 0.905 & $\mathbf{0 . 8 9 4}$ & 0.731 \\
CNN & 0.847 & $\mathbf{0 . 9 3 6}$ & 0.773 & 0.847 & 0.703 \\
RF & 0.846 & 0.850 & $\mathbf{0 . 9 0 8}$ & 0.878 & 0.681 \\
\hline
\end{tabular}

334 As listed in Table 4, the performance in the classification of hit compounds $\left(\mathrm{pIC}_{50} \geq 6\right)$ suggested 335 that LigEGFR performs with the highest balanced accuracy (0.867) and MCC (0.732) compared

336 to the baseline machine learning algorithms. The balanced accuracy is the number of correct

337 predictions divided by the total number of compounds in an imbalanced dataset. Normally, overall

338 accuracy is not a suitable metric for a dataset that is imbalanced [57]. In our test set, all ligands

339 contained 419 active and 280 inactive compounds. MCC is a useful metric to measure performance

340 for binary classification problems. Chicco and Jurman [58] suggested that the MCC works as a

341 truthful metric for the evaluation of binary classification rather than for accuracy and F1-score

342 for imbalanced datasets. Emphasis on overall accuracy and F1-score can be a prevalent mindset

343 leading to mis-interpretation of results, due to lack of consideration of the ratio between positive

344 and negative constituents. In Figure 8a, the ROC plot indicates the classification performance on

345 a different threshold. The result shows that LigEGFR provides AUC at 0.942, which is slightly

346 better than the baseline methods in machine learning $(0.917-0.934)$ and significantly higher

347 than molecular docking techniques $(0.466-0.628)$. Additionally, enrichment plots were used for

348 evaluating how a model can perform in ranking $\mathrm{pIC}_{50}$ value. The enrichment plot was employed

349 by considering the top-ranked data and finding how many active compounds there are. The result

350 showed that the true active compounds, identified by percentages in the LigEGFR model, were

351 mostly higher than others at the same top-ranked dataset percentages (see Figure 8b), which was in

352 an accordance with the ROC plots. 


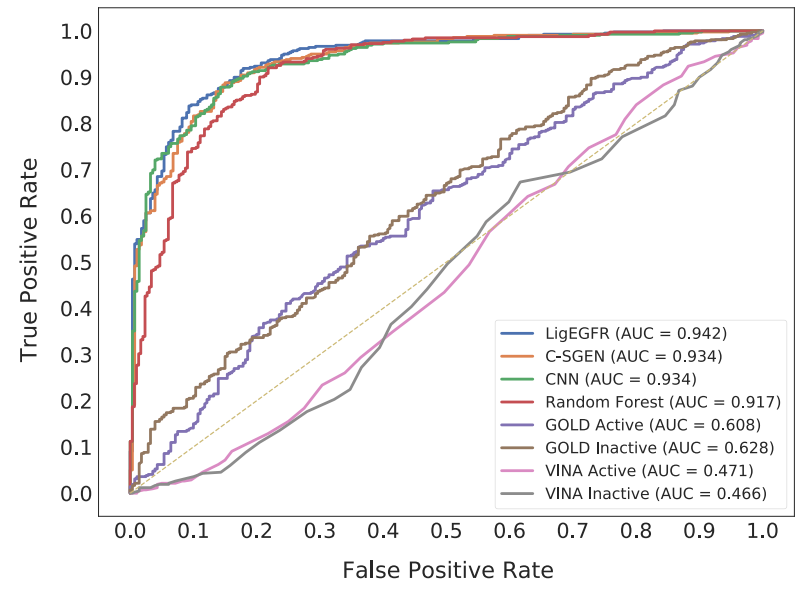

(a) Receiver operating characteristic curve (ROC)

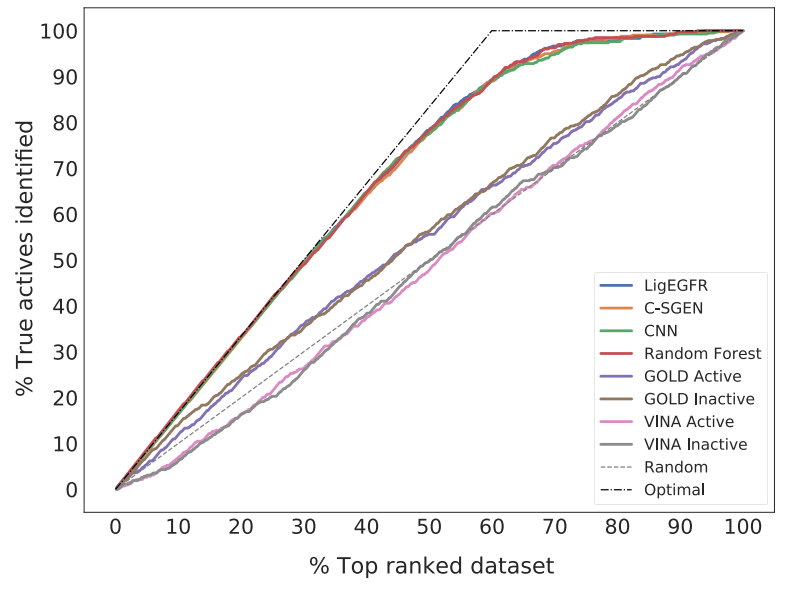

(b) Enrichment plot

Figure 8: Line plots show (a) the receiver operating characteristic curve (ROC) and (b) the enrichment. For the molecular docking approaches, CCDC GOLD and Autodock Vina, were computed with the active and inactive conformations of human EGFR.

353 When comparing baselines between the two techniques, machine learning methods have higher

354 performance than the molecular docking methods for human EGFR. By focusing on molecular 355 docking, we endeavored to achieve ligands of the test set for finding the best orientation within 356 binding pockets of the active and inactive conformations of the human EGFR tyrosine kinase. In

357 general, human EGFR functions in the cell membrane with active and inactive conformations. The 358 active conformation is the asymmetric dimer state that EGFR changes to trans-autophosphorylation 359 of specific tyrosine residues on the kinase domain. As for the inactive state, EGFR can be an 360 apparent the symmetric dimer or monomer, which occurs before phosphorylation [59]. Nevertheless, 361 molecular docking has been persistently undertaken to isolate hit compounds. Wang et al. [55]

362 evaluated the power of docking by ten software packages. The results showed that molecular docking 363 emerged with a weak correlation between docking scores and experimental binding affinities for 364 the PDBbind dataset (version 2014). This failure is because the molecular docking algorithm is 365 conducted from the "lock and key" concept, which does not take account of the real environment.

366 That is to say, it is done without allowing for binding site flexibility and an aqueous solvent 367 [60]. Interestingly, from the Wang et al. report, the flexible docking method, referring to ligand368 protein flexible docking, does not appreciably enhance the correlation between the rank of binding affinity and docking score. In contrast, the semi-flexible (ligand-flexible) docking method has been 
370 recognized to give better correlations than the flexible docking method for both top-scored and best

371 poses. Indeed, the types of docking can assort into rigid docking, semi-flexible docking, and flexible

372 docking [61]. The rigid docking treats protein and ligand as rigid bodies, while semi-flexible and

373 flexible docking methods consider modifications of bond angles, bond lengths, and torsion angles

374 for molecules. However, the docking scoring function, in terms of binding affinity, is least able to

375 distinguish between active and inactive compounds [62].

376 Cumulatively, the contribution of the LigEGFR phenomenon is in line with our expectations. This

377 newly developed model contains the graphical information of the molecules, which is helpful to

378 predict $\mathrm{pIC}_{50}$ prior to hit compound classification. Consequently, our architecture has a better

379 performance for predicting hit compounds among ranked ligands than the existing methods.

\section{Conclusion}

381 Here we have proposed the LigEGFR, a novel deep learning architecture for $\mathrm{pIC}_{50}$ prediction of

382 small molecules against human EGFR. We directly incorporated graphical information of molecules

383 into the embedding layer and trained on the C-SGEN algorithm with architecture adaptation.

384 Additionally, the non-hashed and hashed molecular descriptors have been incorporated in the Conv

385 layers and FC layers, respectively to enhance the predictive performance and generalize the model.

386 The LigEGFR model was trained with various hyperparameters to acquire the optimal model. The

387 experimental evaluations show that LigEGFR has the best performance to predict $\mathrm{pIC}_{50}$. Also,

388 LigEGFR tends to outperform the best existing methods for hit compound classification, with high

389 values of balanced accuracy and MCC. These results were obtained by comparing baseline machine

390 learning algorithms and molecular docking methods.

391 Nowadays, research is focused on machine learning development for $\mathrm{pIC}_{50}$ with the human EGFR

392 target. However, these existing methods have used small-scale datasets, containing less diversity of

393 molecular scaffolds and functional groups. Furthermore, the traditional virtual screening such as

394 molecular docking is insufficient to predict hit compounds by either ranking or classifying the hit

395 compound. LigEGFR was developed to overcome these drawbacks and incorporated training on a 
396 variety of chemical spaces. Moreover, our model exhibits robustness and reliability, confirmed by

397 y-scrambling evaluation and ADAN, respectively.

398 The approach that we declare here opens a new way of applying the drug discovery process via

399 targeted lung cancer therapy. Additionally, this approach can help to avoid disadvantages in virtual

400 screening, reducing computation time, and preventing complications that arise from the off-target

401 discovery of novel hit compounds. Lastly, Python executable script of LigEGFR and web service

402 are freely available at https://github.com/scads-biochem/LigEGFR and https://ligegfr.vistec.ist,

403 respectively.

404

\section{Supplementary data}

405 Supplementary data are available online at https://academic.oup.com/bib.

\section{Code availability and implementation}

407 https://github.com/scads-biochem/LigEGFR

\section{Authors' contribution}

409 P.V., N.SA., B.B., J.K., N.SU., and S.N. conceived and planned the machine learning experiments.

410 P.V., N.SA., and B.B. carried out the machine learning experiments. P.V., N.SA., B.B., R.L., and T.S.

411 performed a web service and executable script. B.B., T.N., and T.R. described cancer and molecular

412 biology concepts. P.V., N.SA., B.B., N.SU., T.R., and S.N. contributed to the interpretation of

413 the results and discussion. P.V., N.SA., and B.B. took the lead in writing the manuscript with

414 the consultation with N.SU., T.R., and S.N. This project has initial ideas for machine learning

415 architecture by P.V., N.SA., B.B., J.K., and S.N.

\section{Conflict of interest}

417 There are no conflicts to declare. 


\section{References}

419 [1] Freddie Bray, Jacques Ferlay, Isabelle Soerjomataram, Rebecca L Siegel, Lindsey A Torre, and Ahmedin Jemal. Global cancer statistics 2018: Globocan estimates of incidence and mortality

[2] Ping Wee and Zhixiang Wang. Epidermal growth factor receptor cell proliferation signaling pathways. Cancers, 9(5):52, 2017.

[3] F Marc Stewart, Hillard M Lazarus, Paul A Levine, Kathy A Stewart, Imad A Tabbara, and Cynthia A Spaulding. High-dose chemotherapy and autologous marrow transplantation for esthesioneuroblastoma and sinonasal undifferentiated carcinoma. American journal of clinical oncology, 12(3):217-221, 1989.

[4] Roy S Herbst, Daniel Morgensztern, and Chris Boshoff. The biology and management of non-small cell lung cancer. Nature, 553(7689):446-454, 2018.

[5] Si-sheng Ou-Yang, Jun-yan Lu, Xiang-qian Kong, Zhong-jie Liang, Cheng Luo, and Hualiang Jiang. Computational drug discovery. Acta Pharmacologica Sinica, 33(9):1131-1140, 2012.

[6] Yu-Chian Chen. Beware of docking! Trends in pharmacological sciences, 36(2):78-95, 2015.

[7] Sunghwan Kim, Jie Chen, Tiejun Cheng, Asta Gindulyte, Jia He, Siqian He, Qingliang Li, Benjamin A Shoemaker, Paul A Thiessen, Bo Yu, et al. Pubchem 2019 update: improved access to chemical data. Nucleic acids research, 47(D1):D1102-D1109, 2019.

[8] David Mendez, Anna Gaulton, A Patrícia Bento, Jon Chambers, Marleen De Veij, Eloy Félix, María Paula Magariños, Juan F Mosquera, Prudence Mutowo, Michał Nowotka, et al. Chembl: towards direct deposition of bioassay data. Nucleic acids research, 47(D1):D930-D940, 2019.

[9] Michael K Gilson, Tiqing Liu, Michael Baitaluk, George Nicola, Linda Hwang, and Jenny Chong. Bindingdb in 2015: a public database for medicinal chemistry, computational chemistry and systems pharmacology. Nucleic acids research, 44(D1):D1045-D1053, 2016.

[10] Markus A Lill. Multi-dimensional qsar in drug discovery. Drug Discovery Today, 12(2324):1013-1017, 2007. 
445 [11] Marta M Stepniewska-Dziubinska, Piotr Zielenkiewicz, and Pawel Siedlecki. Development and evaluation of a deep learning model for protein-ligand binding affinity prediction. Bioinformatics, 34(21):3666-3674, 2018.

[12] Hakime Öztürk, Arzucan Özgür, and Elif Ozkirimli. Deepdta: deep drug-target binding affinity prediction. Bioinformatics, 34(17):i821-i829, 2018.

[13] José Jiménez, Miha Skalic, Gerard Martinez-Rosell, and Gianni De Fabritiis. K deep: Proteinligand absolute binding affinity prediction via 3d-convolutional neural networks. Journal of chemical information and modeling, 58(2):287-296, 2018.

453 [14] Ingoo Lee, Jongsoo Keum, and Hojung Nam. Deepconv-dti: Prediction of drug-target interactions via deep learning with convolution on protein sequences. PLoS computational biology, 15(6):e1007129, 2019.

[15] Izhar Wallach, Michael Dzamba, and Abraham Heifets. Atomnet: a deep convolutional neural network for bioactivity prediction in structure-based drug discovery. arXiv preprint arXiv:1510.02855, 2015.

[16] Jagat Singh Chauhan, Sandeep Kumar Dhanda, Deepak Singla, Subhash M Agarwal, Gajendra PS Raghava, Open Source Drug Discovery Consortium, et al. Qsar-based models for designing quinazoline/imidazothiazoles/pyrazolopyrimidines based inhibitors against wild and mutant egfr. PloS one, 9(7):e101079, 2014.

[17] Hongying Du, Zhide Hu, Andrea Bazzoli, and Yang Zhang. Prediction of inhibitory activity of epidermal growth factor receptor inhibitors using grid search-projection pursuit regression method. PLoS One, 6(7):e22367, 2011.

[18] Shehnaz Fatima and Subhash Mohan Agarwal. Exploring structural features of egfr-her2 dual inhibitors as anti-cancer agents using g-qsar approach. Journal of Receptors and Signal Transduction, 39(3):243-252, 2019.

[19] Silvina E Fioressi, Daniel E Bacelo, and Pablo R Duchowicz. Qsar study of human epidermal growth factor receptor (egfr) inhibitors: conformation-independent models. Medicinal Chemistry Research, 28(11):2079-2087, 2019. 
472 [20] Saw Simeon, Dino Montanari, and Matthew Paul Gleeson. Investigation of factors affecting the performance of in silico volume distribution qsar models for human, rat, mouse, $\operatorname{dog} \&$ monkey. Molecular informatics, 38(10):1900059, 2019.

[21] Aparna Vema, Sunil K Panigrahi, G Rambabu, B Gopalakrishnan, JARP Sarma, and Gautam R Desiraju. Design of egfr kinase inhibitors: a ligand-based approach and its confirmation with structure-based studies. Bioorganic \& medicinal chemistry, 11(21):4643-4653, 2003.

[22] Garima Verma, Mohemmed Faraz Khan, Wasim Akhtar, Mohammad Mumtaz Alam, Mymoona Akhter, Ozair Alam, Syed Misbahul Hasan, and Mohammad Shaquiquzzaman. Pharmacophore modeling, 3d-qsar, docking and adme prediction of quinazoline based egfr inhibitors. Arabian Journal of Chemistry, 12(8):4815-4839, 2019.

[23] Xiaofeng Wang, Zhen Li, Mingjian Jiang, Shuang Wang, Shugang Zhang, and Zhiqiang Wei. Molecule property prediction based on spatial graph embedding. Journal of chemical information and modeling, 59(9):3817-3828, 2019.

[24] Adam Paszke, Sam Gross, Francisco Massa, Adam Lerer, James Bradbury, Gregory Chanan, Trevor Killeen, Zeming Lin, Natalia Gimelshein, Luca Antiga, et al. Pytorch: An imperative

[25] Bharath Ramsundar, Peter Eastman, Patrick Walters, Vijay Pande, Karl Leswing, and Zhenqin style, high-performance deep learning library. In Advances in neural information processing Wu. Deep Learning for the Life Sciences. O’Reilly Media, 2019.

[26] Reaxys. Reaxys medicinal chemistry. https://www. reaxys.com.

[27] Wes McKinney et al. Data structures for statistical computing in python. In Proceedings of the 9th Python in Science Conference, volume 445, pages 51-56. Austin, TX, 2010.

[28] Stéfan van der Walt, S Chris Colbert, and Gael Varoquaux. The numpy array: a structure for efficient numerical computation. Computing in science \& engineering, 13(2):22-30, 2011. 17(3):261-272, 2020. 
500

[30] John D Hunter. Matplotlib: A 2d graphics environment. Computing in science \& engineering, 9(3):90-95, 2007.

[31] Fabian Pedregosa, Gaël Varoquaux, Alexandre Gramfort, Vincent Michel, Bertrand Thirion, Olivier Grisel, Mathieu Blondel, Peter Prettenhofer, Ron Weiss, Vincent Dubourg, et al. Scikitlearn: Machine learning in python. the Journal of machine Learning research, 12:2825-2830, 2011.

[32] G Landrum. Rdkit: Open-source cheminformatics software. GitHub and SourceForge, $10: 3592822,2016$.

[33] Charles Tapley Hoyt, Andrej Konotopez, and Christian Ebeling. Pybel: a computational framework for biological expression language. Bioinformatics, 34(4):703-704, 2018.

[34] Michael Waskom, Olga Botvinnik, Joel Ostblom, Maoz Gelbart, Saulius Lukauskas, Paul Hobson, David C Gemperline, Tom Augspurger, Yaroslav Halchenko, John B Cole, et al. mwaskom/seaborn: v0. 10.1 (april 2020). Zenodo, 2020.

[35] Egon L Willighagen, John W Mayfield, Jonathan Alvarsson, Arvid Berg, Lars Carlsson, Nina Jeliazkova, Stefan Kuhn, Tomáš Pluskal, Miquel Rojas-Chertó, Ola Spjuth, et al. The chemistry development kit (cdk) v2. 0: atom typing, depiction, molecular formulas, and substructure searching. Journal of cheminformatics, 9(1):33, 2017.

[36] Thomas N Kipf and Max Welling. Semi-supervised classification with graph convolutional networks. arXiv preprint arXiv:1609.02907, 2016.

[37] Christoph Rücker, Gerta Rücker, and Markus Meringer. y-randomization and its variants in qspr/qsar. Journal of chemical information and modeling, 47(6):2345-2357, 2007.

[38] Johann Gasteiger. Handbook of chemoinformatics. Wiley-VCH, 2003.

[39] Tatiana I Netzeva, Andrew P Worth, Tom Aldenberg, Romualdo Benigni, Mark TD Cronin, Paola Gramatica, Joanna S Jaworska, Scott Kahn, Gilles Klopman, Carol A Marchant, et al. Current status of methods for defining the applicability domain of (quantitative) structureactivity relationships: The report and recommendations of ecvam workshop 52. Alternatives to Laboratory Animals, 33(2):155-173, 2005. 
527 [40] Faizan Sahigara, Kamel Mansouri, Davide Ballabio, Andrea Mauri, Viviana Consonni, and Roberto Todeschini. Comparison of different approaches to define the applicability domain of qsar models. Molecules, 17(5):4791-4810, 2012.

530 [41] Alexander Tropsha. Best practices for qsar model development, validation, and exploitation. Molecular informatics, 29(6-7):476-488, 2010.

[42] Jang-Sik Choi, My Kieu Ha, Tung Xuan Trinh, Tae Hyun Yoon, and Hyung-Gi Byun. Towards a generalized toxicity prediction model for oxide nanomaterials using integrated data from different sources. Scientific reports, 8(1):1-10, 2018.

[43] Nina Nikolova-Jeliazkova and Joanna Jaworska. An approach to determining applicability domains for qsar group contribution models: an analysis of src kowwin. Alternatives to Laboratory Animals, 33(5):461-470, 2005.

[44] Michael M Mysinger, Michael Carchia, John J Irwin, and Brian K Shoichet. Directory of useful decoys, enhanced (dud-e): better ligands and decoys for better benchmarking. Journal

[45] Nicolas Triballeau, Francine Acher, Isabelle Brabet, Jean-Philippe Pin, and Hugues-Olivier Bertrand. Virtual screening workflow development guided by the "receiver operating characteristic" curve approach. application to high-throughput docking on metabotropic glutamate of medicinal chemistry, 55(14):6582-6594, 2012. receptor subtype 4. Journal of medicinal chemistry, 48(7):2534-2547, 2005.

[46] Kathleen Aertgeerts, Robert Skene, Jason Yano, Bi-Ching Sang, Hua Zou, Gyorgy Snell, Andy Jennings, Keiji Iwamoto, Noriyuki Habuka, Aki Hirokawa, et al. Structural analysis of the

[47] Edgar R Wood, Anne T Truesdale, Octerloney B McDonald, Derek Yuan, Anne Hassell, mechanism of inhibition and allosteric activation of the kinase domain of her2 protein. Journal Scott H Dickerson, Byron Ellis, Christopher Pennisi, Earnest Horne, Karen Lackey, et al. A unique structure for epidermal growth factor receptor bound to gw572016 (lapatinib): relationships among protein conformation, inhibitor off-rate, and receptor activity in tumor cells. Cancer research, 64(18):6652-6659, 2004. 
[48] Andrew Leaver-Fay, Michael Tyka, Steven M Lewis, Oliver F Lange, James Thompson, Ron Jacak, Kristian W Kaufman, P Douglas Renfrew, Colin A Smith, Will Sheffler, et al. Rosetta3: an object-oriented software suite for the simulation and design of macromolecules. In Methods in enzymology, volume 487, pages 545-574. Elsevier, 2011.

[49] Gareth Jones, Peter Willett, Robert C Glen, Andrew R Leach, and Robin Taylor. Development and validation of a genetic algorithm for flexible docking. Journal of molecular biology, 267(3):727-748, 1997.

[50] Oliver Korb, Thomas Stutzle, and Thomas E Exner. Empirical scoring functions for advanced protein- ligand docking with plants. Journal of chemical information and modeling, 49(1):8496, 2009.

[51] Oleg Trott and Arthur J Olson. Autodock vina: improving the speed and accuracy of docking with a new scoring function, efficient optimization, and multithreading. Journal of computational chemistry, 31(2):455-461, 2010.

[52] Alexander Golbraikh, Min Shen, Zhiyan Xiao, Yun-De Xiao, Kuo-Hsiung Lee, and Alexander Tropsha. Rational selection of training and test sets for the development of validated qsar models. Journal of computer-aided molecular design, 17(2-4):241-253, 2003.

[53] Vladimir Svetnik, Andy Liaw, Christopher Tong, J Christopher Culberson, Robert P Sheridan, and Bradley P Feuston. Random forest: a classification and regression tool for compound classification and qsar modeling. Journal of chemical information and computer sciences, 43(6):1947-1958, 2003.

[54] Svante Wold, Michael Sjöström, and Lennart Eriksson. Pls-regression: a basic tool of chemometrics. Chemometrics and intelligent laboratory systems, 58(2):109-130, 2001.

[55] Zhe Wang, Huiyong Sun, Xiaojun Yao, Dan Li, Lei Xu, Youyong Li, Sheng Tian, and Tingjun Hou. Comprehensive evaluation of ten docking programs on a diverse set of protein-ligand complexes: the prediction accuracy of sampling power and scoring power. Physical Chemistry Chemical Physics, 18(18):12964-12975, 2016.

[56] Dariusz Plewczynski, Michał Łaźniewski, Rafał Augustyniak, and Krzysztof Ginalski. Can we trust docking results? evaluation of seven commonly used programs on pdbbind database. 
Journal of computational chemistry, 32(4):742-755, 2011.

583 [57] Selcuk Korkmaz. Deep learning-based imbalanced data classification for drug discovery.

$584 \quad$ Journal of Chemical Information and Modeling, 60(9):4180-4190, 2020.

585 [58] Davide Chicco and Giuseppe Jurman. The advantages of the matthews correlation coefficient (mcc) over f1 score and accuracy in binary classification evaluation. BMC genomics, 21(1):6, 2020.

588 [59] Ruth Nussinov, Hyunbum Jang, Chung-Jung Tsai, and Feixiong Cheng. Precision medicine and driver mutations: Computational methods, functional assays and conformational principles for interpreting cancer drivers. PLoS computational biology, 15(3):e1006658, 2019.

591 [60] Pedro J Ballester, Adrian Schreyer, and Tom L Blundell. Does a more precise chemical description of protein-ligand complexes lead to more accurate prediction of binding affinity? Journal of chemical information and modeling, 54(3):944-955, 2014.

594 [61] Francesca Tessaro and Leonardo Scapozza. How 'protein-docking'translates into the new emerging field of docking small molecules to nucleic acids? Molecules, 25(12):2749, 2020.

596 [62] J. Cole, E. Davis, G. Jones, and C.R. Sage. 3.12 - molecular docking - a solved problem? In Samuel Chackalamannil, David Rotella, and Simon E. Ward, editors, Comprehensive Medicinal Chemistry III, pages 297 - 318. Elsevier, Oxford, 2017. 